\title{
Avaliação dos valores de lactato e da atividade sérica da enzima creatina quinase (2.7.3.2) em cavalos (Equus caballus) da raça Puro-Sangue-Inglês (PSI) submetidos a teste de esforço em esteira ergométrica
}

\section{Evaluation of plasma lactate and seric activity of creatine kinase values in thoroughbred (PSI) horses (Equus caballus) submitted to effort tests on treadmill}

\author{
Rosalie Joslin Kowal, ${ }^{*}$ Nádia Regina Pereira Almosny,, Bianca Cascardo, ${ }^{* * *}$ \\ Ricardo Peixoto Summa, ${ }^{* * * *}$ Leopoldo José Cury ${ }^{\star * \star * *}$
}

\begin{abstract}
Resumo
O Puro-Sangue-Inglês (PSI) encontra-se difundido em todo o Brasil e dada a falta de dados sobre a fisiologia do exercício destes animais, torna-se importante a realização de testes de esforço sob algumas condições características visando a obter informações mais precisas e adequadas quando esses cavalos são submetidos a esforços constantes. Logo, decidiu-se investigar as causas de baixa e de perda de performance nos cavalos de corridas, utilizando-se esteira ergométrica. Foram utilizados 20 animais em fase de treinamento no Jockey Club Brasileiro, com idade entre 2 e 6 anos, machos e fêmeas, submetidos a testes de esforço em esteira visando avaliar dados bioquímicos - lactato plasmático e creatina quinase (CK). Os valores médios variaram nos tempos estudados e foram correlacionados e avaliados estatisticamente. Concluiu-se que a realização de teste de esforço em esteira ergométrica é útil para a determinação destes parâmetros e tem como principal função avaliar a capacidade atlética de cavalos de corridas, bem como detectar possíveis momentos de baixa performance permitindo a sua correção.
\end{abstract}

Palavras-chave: eqüino, esteira, exercício, lactato, creatina quinase.

\begin{abstract}
Thoroughbred horses are spread in Brazil and due to the lack of data concerning their exercise physiology, it is important to do exercise tests under some characteristic conditions in order to obtain precise and proper data as this horses are submitted to constant efforts. Thus, it was decided to investigate the causes of poor performance in racehorses, using a treadmill. Twenty Thoroughbred racehorses in training at Jockey Club Brasileiro, with age ranging from 2 to 6 years old, males and females, submitted to effort tests on treadmill in order to evaluate biochemical (plasma lactate and CK concentrations) values. The mean values varied at the studied times and they were statistically evaluated and correlated. It was concluded that the exercise tests on a treadmill are useful for the determination of this parameters and they have as main role to evaluate the athletic ability of racehorses, as well as to detect possible poor performance times, allowing their correction.
\end{abstract}

Keywords: equine, treadmill, exercise, lactate, creatina kinase.

\section{Introdução}

O cavalo (Equus caballus) encontra-se hoje amplamente difundido no mundo e dada à falta de dados sobre a fisiologia do exercício destes animais em nosso país, torna-se importante a realização de testes de esforço visando a obter informações mais precisas e adequadas dos nossos cavalos quando submetidos a esforços constantes.

Durante o exercício de alta velocidade as células somente podem manter o suprimento adequado de adenosina trifosfato (ATP) às células musculares pelo uso anaeróbico da glicose

\footnotetext{
* Veterinária do Jockey Club Brasileiro.

** Prof ${ }^{a}$. Dr ${ }^{a}$. do Departamneto de Clínica e Patologia Clínica da Universidade Federal Fluminense.

*** Veterinária autônoma do Jockey Club Brasileiro.

**** Veterinário autônomo.

${ }^{* * * *}$ Veterinário autônomo do Jockey Club Brasileiro e Prof. de Reprodução Animal da Universidade Federal Fluminense.
} 
(Eaton, 1994). Então, sob essas condições anaeróbicas, o ácido lático é produzido pela descarboxilação do piruvato, tendo como catalisador da reação a enzima lactato desidrogenase (LDH). (Luna, 2002).

Lactato: o ácido lático formado é rapidamente tamponado em parte pelo bicarbonato extracelular, resultando na produção de lactato (Rose e Post, 2001).

Esse aumento da concentração de lactato poderá ser usado para indicar a

capacidade atlética do cavalo (Rose ${ }^{A}$, s.d.; Coffman, 1982; Rose e Hodgson, 1994, Rose e Post, 2001; Rainger et al., 1994; Rainger et al., 1995).

Animais que apresentam grande capacidade aeróbica geralmente têm baixas concentrações de lactato em resposta ao exercício ou apresentam uma clearance mais eficiente (Eaton et al., 1999; Valette et al., 1993; Rose e Hodgson, 1994;).

Existe correlação positiva entre o aumento da concentração de lactato no sangue e a intensidade do exercício (Davie e Evans, 2000; Misumi et al., 1994).

A relação lactato $X$ performance é determinada pela VLa4, velocidade na qual a concentração de lactato é igual a $4 \mathrm{mmol} /$ L (Eaton et al., 1999), sendo utilizada para comparar o potencial atlético dos animais (Rose e Hodgson, 1994; Rainger et al., 1995) (Figura 1).

O metabolismo do lactato varia entre os indivíduos, mas para a maioria dos cavalos o retorno aos valores próximos aos níveis basais ocorre em aproximadamente 1 hora após 0 término do exercício (Rose ${ }^{\mathrm{A}}$, s.d.).

O lactato se acumula no sangue em função da velocidade do exercício e tem sua concentração bastante elevada quando próxima do final do exercício, indicando grande contribuição da glicólise anaeróbica para produção de energia (Morris, 1992; Davie e Evans, 2000).

Enzimas musculares: condições clínicas como a rabdomiólise podem ser diagnosticadas pela medida da atividade de algumas enzimas liberadas pelos músculos lesados. As enzimas aspartato aminotranferase (AST), creatina quinase (CK) e lactato desidrogenase (LDH) têm sua concentração aumentada no sangue e são as mais utilizadas para avaliação do sistema muscular (Rose, s.d.; Harris et al., 1998).

Alguns indivíduos podem apresentar atividade sérica elevada de CK, bem como de AST, sem apresentar sinais de lesões musculares, mas a tendência é que, com o treinamento, a incidência destes achados diminua (Cardinet et al., 1963; Harris et al., 1998).

A adoção de novas técnicas de treinamento e acompanhamento das atividades enzimáticas na rotina de cavalos em treinamento deve auxiliar na avaliação da performance, diminuindo a ocorrência de lesões ou de fadiga muscular (Evans, 2000).

Assim, o objetivo do presente trabalho foi avaliar os valores de lactato e da atividade sérica da enzima creatina quinase em cavalos da raça Puro Sangue Inglês (PSI) submetidos a teste de esforço em esteira ergométrica.

\section{Material e métodos}

Foram utilizados neste trabalho 20 animais da raça PuroSangue-Inglês (PSI), na faixa etária de 2 a 6 anos, machos e fêmeas, em fase de treinamento no Jockey Club Brasileiro. Esse treinamento é de responsabilidade do treinador do animal, sendo por ele determinado.

Em todos os animais foram realizados exames clínicos rotineiros, em repouso, e avaliação do histórico do seu desempenho atlético. Todos os animais apresentaram boas condições físicas, sem quaisquer problemas aparentes. A principal reclamação dos treinadores quanto à performance dos animais era o baixo desempenho atlético. Todos os animais foram submetidos a um período de adaptação ao exercício na esteira ergométrica GALLOPER 5500. O programa de adaptação levou de cinco a sete dias, consistindo desde o simples reconhecimento do animal à esteira até o galope confortável. Nesta fase foram registrados o batimento cardíaco e o comportamento dos animais nas diferentes velocidades a que foram submetidos. Alguns animais não se adaptaram à esteira e por isso não foram utilizados no trabalho.

O seguinte esquema foi adotado:

Inclinação de 6 graus, em relação ao solo, na esteira: 4 minutos a $2 \mathrm{~m} / \mathrm{s}-3$ minutos a $4 \mathrm{~m} / \mathrm{s}-2$ minutos a $6 \mathrm{~m} / \mathrm{s}-1$ minuto a $8 \mathrm{~m} / \mathrm{s}-3$ minutos a $4 \mathrm{~m} / \mathrm{s}-1$ minuto a $2 \mathrm{~m} / \mathrm{s}$.

Uma vez estando o animal apto a realizar o teste de esforço, seguiu-se o procedimento adiante descrito:

1) Foram realizadas coletas de amostras de sangue na cocheira, com o animal em repouso, em tubos de coleta a vácuo ${ }^{1} \mathrm{com}$ heparina sódica e sem anticoagulante. As amostras eram imediatamente armazenadas em recipiente isotérmico e levadas em seguida ao laboratório do Hospital Octávio Dupont, onde eram realizadas as análises.

2) A preparação do animal para a realização do teste de esforço consistiu de: a) tricotomia do local onde se situa topograficamente a veia jugular direita; b) rigorosa assepsia; c) anestesia do local da punção venosa com lidocaína $2 \%$ sem vasoconstritor, ${ }^{2}$ d) punção venosa e instalação de catéter ${ }^{3}$ e sistema extensor; e) fixação do catéter na pele com fio Vicryl 2.0, ${ }^{4}$ f) heparinização (solução de cloreto de sódio a $0,9 \%{ }^{5}$ e heparina sódica ${ }^{6}$ ), do sistema coletor de amostras.

3) O animal então era conduzido à esteira ergométrica, davase a inclinação de 6 graus, para simular condições similares àquelas estabelecidas em condições de corridas, como compensar a ausência do peso do jóquei, e dava-se início ao exercício (Figura 2).

4) As velocidades impostas e amostras de sangue coletadas para as análises se deram da seguinte forma:

- coleta de amostra para análise de lactato (tubos com heparina) e CK (tubos sem anticoagulante) no momento repouso.

\footnotetext{
${ }^{1}$ Tubos para coleta de sangue a vácuo - Vacutainer BD

${ }^{2}$ Cloridrato de lidocaína $2 \%$ sem vasoconstritor - Xilestein 2\% - Crystalia

${ }^{3}$ Cateter Intracath $12 \mathrm{G}$ BD

${ }^{4}$ Fio Vicryl 2.0 - Ethicon

${ }^{5}$ Solução de cloreto de sódio 0,9\% - Fresenius Kabi

${ }^{6}$ Heparina Sódica - Liquemine - Roche - 25.000 UI
} 
- em todas as velocidades do exercício foram realizadas coletas para análise da concentração do lactato. As velocidades do teste de esforço foram as seguintes: $2 \mathrm{~m} /$ $\mathrm{s}-2 \mathrm{~min}$ (passo), 3m/s-4 min (trote), $4 \mathrm{~m} / \mathrm{s}-1 \mathrm{~min}$ (galope), $5 \mathrm{~m} / \mathrm{s}-1$ min e, assim sucessivamente, até a máxima velocidade desenvolvida pelo animal.

- coleta de amostra para análise de lactato e CK no momento fim do exercício.

- coleta de amostra para análise de lactato nos momentos 5, 10, 15 e 20 minutos após o término do exercício.

- coleta de amostra para análise de lactato e CK nos momentos 2, 6 e 24 horas após o término do exercício.

Faltando 15 segundos para cada troca de velocidade, dava-se início à coleta da amostra. Primeiramente coletando-se $40 \mathrm{ml}$ que seriam descartados por estarem diluídos com a solução heparinizada e, posteriormente, coletando-se os $10 \mathrm{ml}$ da amostra a ser avaliada. Para isso foram utilizadas seringas de 60 e de $20 \mathrm{ml},{ }^{7}$ respectivamente. O sangue coletado nas seringas de $20 \mathrm{ml}$ era transferido para os tubos Vacutainer com heparina sódica. As seringas de $20 \mathrm{ml}$ foram desprezadas após serem utilizadas. Imediatamente após a coleta, o sistema de cateter e extensor eram heparinizados. O teste de esforço seguiu até a velocidade máxima que o animal conseguiu desenvolver, mesmo com todo incentivo da equipe. As velocidades máximas obtidas variaram de $10-12$ $\mathrm{m} / \mathrm{s}$, sendo que 1 animal atingiu somente até $9 \mathrm{~m} / \mathrm{s}$.

Seguiu-se a coleta das amostras nos momentos 5, 10, 15 e 20 minutos após o exercício, com o animal ainda na esteira, em velocidade de passo ou já fora da esteira, mas caminhando na área próxima do exercício.

Nos momentos 2, 6 e 24 horas após o teste de esforço, eram coletadas amostras para a análise bioquímica, estando o animal na cocheira, em repouso.

As amostras foram centrifugadas, quando necessário, e analisadas no aparelho: EKTACHEM DT60 II System da Johnson e Johnson.

Em todos os momentos acompanhados foram observadas e anotadas as freqüências cardíacas dos animais, sendo importante para este trabalho as frequências nos instantes finais do teste de esforço, para determinação do seu término. A mínima freqüência observada na fase final do exercício foi de 205 bpm e a máxima foi de 237 bpm.

O tempo de permanência do animal na esteira durante o teste de esforço variou de 14 a 18 minutos, dependendo da velocidade atingida pelo animal no seu máximo esforço físico.

\section{Delineamento estatístico}

Os dados laboratoriais foram analisados estatisticamente por método não paramétrico de Wilcoxon com nível de significância de 5\% (Rodrigues, 1993).

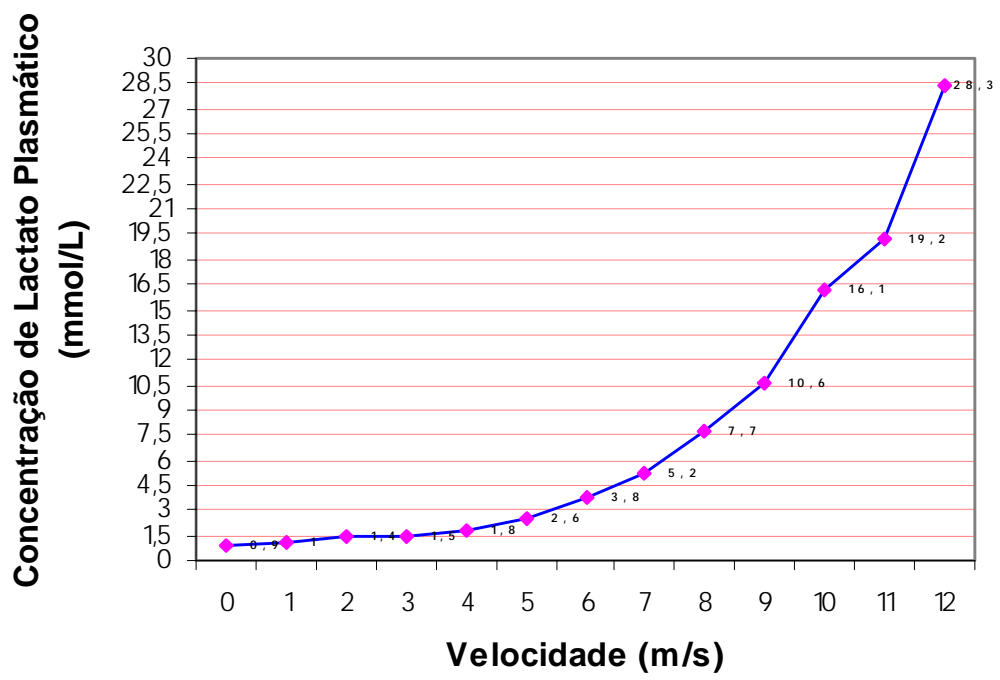

Figura 1: Gráfico que representa a relação entre o aumento da concentração de lactato $(\mathrm{mmol} / \mathrm{L})$ e aumento da velocidade $(\mathrm{m} / \mathrm{s})$ durante realização do teste de esforço.

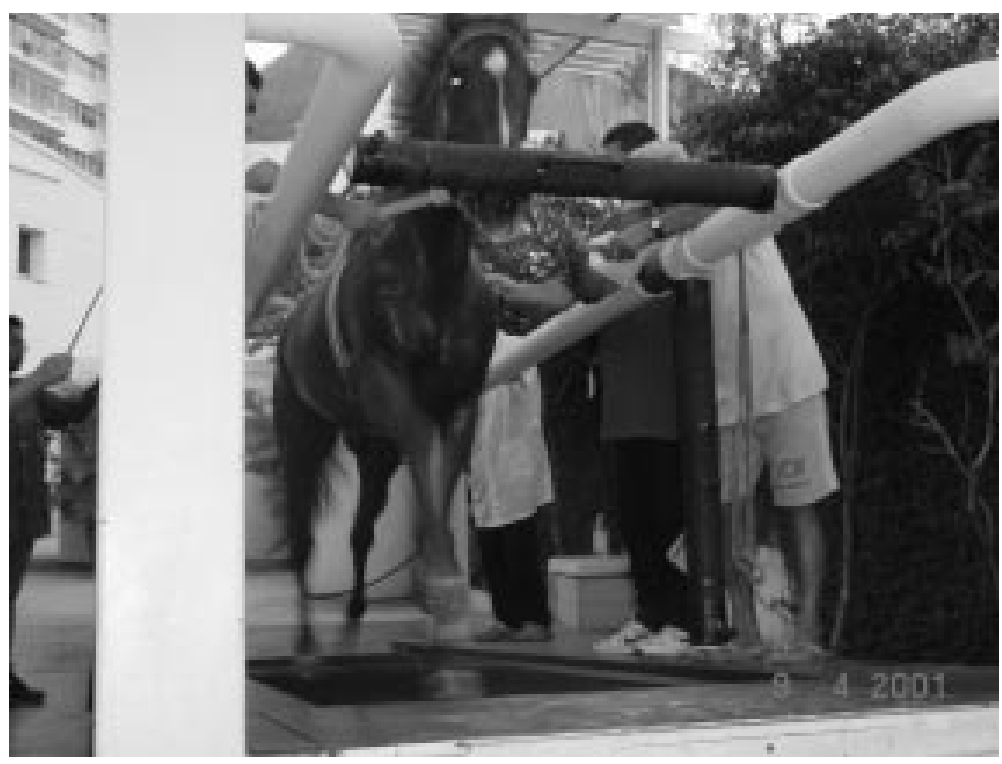

Figura 2: Animal em galope $(8 \mathrm{~m} / \mathrm{s})$ em esteira ergométrica durante o teste de esforço.

7 Seringas de 10, 20 e $60 \mathrm{ml}$ Plastipak BD 


\section{Resultados e discussão}

Tabela 1: VALORES DE LACTATO $(\mathrm{mmol} / \mathrm{L})$ de eqüinos submetidos a teste de esforço em esteira ergométrica: em repouso (Lactato-0); nas velocidades de: $2 \mathrm{~m} / \mathrm{s}$ (Lactato-2); $3 \mathrm{~m} / \mathrm{s}$ (Lactato-3); 4m/s (Lactato-4:); 5m/s (Lactato-5); 6m/s (Lactato-6:); 7m/s (Lactato-7); 8m/s (Lactato-8:); 9m/s (Lactato-9:) ;10m/s (Lactato-10:); 11m/s (Lactato-11); 12m/s (Lactato-12:); no momento depois do exercício (Lactato-D); 5 min. depois do exercício (Lactato-5D:) ;10 min. depois do exercício (Lactato-10D); $15 \mathrm{~min}$. depois do exercício (Lactato-15D); $20 \mathrm{~min}$. depois do exercício (Lactato20D); 2 hs. depois do exercício (Lactato-D2H); 6hs. depois do exercício (Lactato-D6H:) e 24 horas depois do exercício (Lactato-D24H).

\begin{tabular}{l|l|l|c|c|c}
\hline & $\mathbf{n}$ & MÉDIA & DESVIO-PADRÃO & VALOR MÍNIMO & VALOR MÁXIMO \\
\hline LACTATO-0 & 20 & 0,975 & 0,335 & 0,60 & 1,80 \\
\hline LACTATO-2 & 20 & 1,430 & 0,695 & 0,60 & 3,20 \\
\hline LACTATO-3 & 20 & 1,515 & 0,885 & 0,60 & 4,50 \\
\hline LACTATO-4 & 20 & 1,810 & 0,721 & 0,90 & 3,90 \\
\hline LACTATO-5 & 20 & 2,665 & 0,938 & 1,60 & 6,60 \\
\hline LACTATO-6 & 20 & 3,820 & 1,152 & 2,00 & 7,90 \\
\hline LACTATO-7 & 20 & 5,255 & 1,384 & 3,30 & 11,70 \\
\hline LACTATO-8 & 20 & 7,700 & 1,933 & 5,30 & 14,00 \\
\hline LACTATO-9 & 20 & 10,605 & 1,653 & 8,00 & 28,00 \\
\hline LACTATO-10 & 17 & 16,117 & 4,456 & 9,00 & 35,00 \\
\hline LACTATO-11 & 11 & 19,272 & 6,604 & 11,00 & 71,00 \\
\hline LACTATO-12 & 3 & 28,333 & 7,371 & 20,00 & 50,00 \\
\hline LACTATO-D & 20 & 28,900 & 13,844 & 9,00 & 60,00 \\
\hline LACTATO-5D & 19 & 25,421 & 11,137 & 6,00 & 37,00 \\
\hline LACTATO-10D & 19 & 25,789 & 14,156 & 3,90 & 53,00 \\
\hline LACTATO-15D & 19 & 18,873 & 9,264 & 3,50 & 3,40 \\
\hline LACTATO-20D & 19 & 16,473 & 12,603 & 0,70 & 1,90 \\
\hline LACTATO-D2H & 18 & 1,600 & 0,726 & 0,60 & 1,60 \\
\hline LACTATO-D6H & 20 & 1,045 & 0,367 & 0,60 & \\
\hline LACTATO-D24H & 20 & 0,955 & 0,296 & & \\
\hline
\end{tabular}

$\mathrm{n}$ : número de animais avaliados.

Tabela 2: VALORES DE VLa4 (m/s); Concentração de lactato (mmol/l) imediatamente e 2 horas após ao exercício, de equinos submetidos a teste de esforço em esteira ergométrica.

\begin{tabular}{c|c|c|c}
\hline Animal & VLa4 (m/s) & $\begin{array}{c}\text { Concentração de lactato depois } \\
\text { do exercício (mmol/l) }\end{array}$ & $\begin{array}{c}\text { Concentração de lactato 2 horas } \\
\text { depois do exercício (mmol/l) }\end{array}$ \\
\hline $\mathbf{1}$ & $6-7$ & 28 & 1,4 \\
\hline $\mathbf{2}$ & $7-8$ & 36 & $0,9(6 \mathrm{hs})$ \\
\hline $\mathbf{3}$ & $6-7$ & 46 & 1,4 \\
\hline $\mathbf{4}$ & $5-6$ & 38 & 2,4 \\
\hline $\mathbf{5}$ & $5-6$ & 31 & 3 \\
\hline $\mathbf{6}$ & $6-7$ & 71 & 1,9 \\
\hline $\mathbf{7}$ & $7-8$ & 40 & $1,2(6 \mathrm{hs})$ \\
\hline $\mathbf{8}$ & 6 & 42 & 3,4 \\
\hline $\mathbf{9}$ & $6-7$ & 25 & 1,7 \\
\hline $\mathbf{1 0}$ & $6-7$ & 19 & 1 \\
\hline $\mathbf{1 1}$ & $7-8$ & 25 & 1,5 \\
\hline $\mathbf{1 2}$ & $5-6$ & 22 & 1,2 \\
\hline $\mathbf{1 3}$ & $5-6$ & 13 & 1,2 \\
\hline $\mathbf{1 4}$ & $4-5$ & 24 & 1,6 \\
\hline $\mathbf{1 5}$ & $5-6$ & 15 & 0,7 \\
\hline $\mathbf{1 6}$ & $5-6$ & 16 & 2 \\
\hline $\mathbf{1 7}$ & $5-6$ & 28 & 1,2 \\
\hline $\mathbf{1 8}$ & $7-8$ & 21 & 1,5 \\
\hline $\mathbf{1 9}$ & $6-7$ & 25 & 0,9 \\
\hline $\mathbf{2 0}$ & $5-6$ & 13 & 0,8 \\
\hline
\end{tabular}


O aumento da concentração de lactato conseqüente ao aumento da intensidade do exercício esteve de acordo com os trabalhos de Valette et al. (1993), Rainger et al. (1995), Geor et al. (1999), Fenger et al. (2000) e Manohar et al. (2001) que trabalharam com animais exercitados em esteira. Esta elevação foi relacionada com o aumento da atividade muscular (Mayes, 1998) e isto foi confirmado pela elevação de cerca de $50 \%$ da atividade sérica de CK.

O aumento da concentração de lactato (VLa4), indicando maior utilização do metabolismo anaeróbico para produção de energia, ocorreu nas velocidades de 5 a $6 \mathrm{~m} / \mathrm{s}$, com as maiores concentrações imediatamente após o término do exercício para a maioria dos animais, concordando com o trabalho de Seeherman e Morris (1990) (Figura 2). Para três animais, a maior concentração foi observada 5 minutos após o término do exercício e para dois animais ocorreu em 10 minutos após o término do teste.

Para aqueles animais que apresentaram maiores concentrações de lactato no momento depois do término do teste, em 5-10 minutos a concentração de lactato começou a apresentar redução de seus valores, concordando com Evans et al. (1993), que observou queda da concentração de lactato em 2-5 minutos após o término do exercício.

Redução mais acentuada da concentração de lactato foi observada em 15-20 minutos após o término do exercício. Após duas horas os níveis de lactato no plasma se encontraram bem próximos dos valores basais, assim como nos momentos 6 e 24 horas após.

O VLa4 variou para os animais submetidos ao teste, sendo maior para aqueles de maior performance, o que está de acordo com Couroucé et al. (1997).

A maior concentração de lactato plasmático medido ao final de cada velocidade aumentou em relação à medida anterior, que concorda com o trabalho de Harris et al. (1998).

Para todos os momentos houve diferença significativa ( $p$ valor $<0,05$ ) do lactato com o momento anterior, com exceção dos momentos 2-3, 11-12, 12-D, D-D5, D5-D10 e D6H-D24H.
Concentração de CK ( UI )

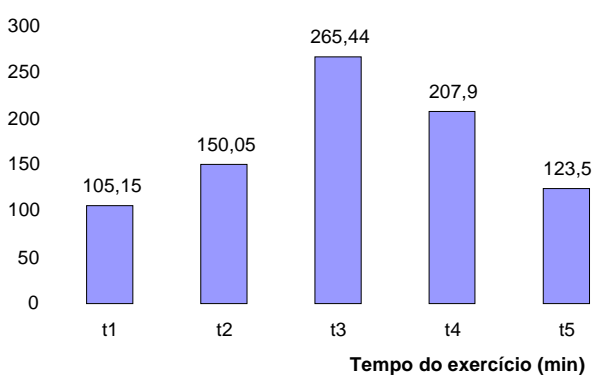

Figura 3: Gráfico que representa a atividade sérica da enzima creatina quinase (CK) no momento repouso e nos momentos seguintes ao término do exercício.

Onde: t1: momento repouso

t2: momento logo após o término do exercício

t3: 2 horas após o término do exercício

t4: 6 horas após o término do exercício

t5: 24 horas após o término do exercício

As atividades séricas da enzima creatina quinase (CK) apresentaram concentrações acima dos valores basais após o término do exercício e continuou aumentando até o momento 2 horas depois do término do teste de esforço. Seis horas após a sua concentração plasmática já havia diminuído um pouco e, em 24 horas após o exercício, a concentração plasmática já se encontrava próxima aos valores observados no momento repouso. Isto ocorreu porque foi respeitado o início da fadiga muscular dos animais, que não foram submetidos, portanto, a um teste de esforço que excedesse a sua capacidade máxima de resposta ao exercício (Figura 3).

Tyler-McGowan et al. (1999) observaram que o treinamento pode levar a adaptações músculo-esqueléticas, mas elevações das atividades de AST e CK podem aparecer associadas a dor muscular, fadiga e baixa performance. Segundo Toledo et al. (2001) ocorre aumento da atividade sérica de CK após exercícios intensos.

$O$ aumento da atividade sérica de CK foi relacionado com a permeabilidade de membrana aumentada devido à hipóxia que se desenvolve durante o exercício. Animais mal condicionados fisicamente apresentarão hipóxia em trabaIhos de baixas intensidades e, conseqüentemente, maiores concentrações da enzima em relação àqueles animais bem condiciona-

Para todos os momentos houve diferença significativa ( $p$ valor $<0,05)$ da enzima creatina quinase $(\mathrm{CK})$ com o momento anterior, com exceção de D2H-D6H. dos, conforme também observaram Milne (1976); Snow e Mackenzie (1977); Milne (1982) e Seeherman e Morris (1990). 


\section{Conclusão e sugestões}

O teste de esforço em esteira ergométrica permite a determinação de valores bioquímicos, valores esses de extrema importância na avaliação da capacidade atlética desejável. Tal capacidade é compreendida como a integração dos vários sistemas corporais, os quais determinam a produção de energia despendida em uma disputa.

A análise destes parâmetros, obtidos nos mesmos animais em duas fases diferentes, antes e após treinamento com intensidade submáxima, na velocidade em que o animal atinge o início da produção anaeróbica de energia (VLa4), pode ser um indicativo confiável para a avaliação do desempenho atlético de um animal.

A avaliação da concentração sérica do lactato é importante para a definição da participação do metabolismo anaeróbico na produção de energia que será utilizada pelo animal durante um exercício intenso e de curta duração.

\section{Referências}

CARDINET, G. H.; FOWLER, M. E.; TYLER, W. S. The Effects of Training, Exercise and Tying-Up on Serum Transaminase Activities in the Horse. American Journal of Veterinary Research. v. 24, n. 3, p. 980-985, 1963. COFFMAN, J. R. Metabolism and Enzimology in Equine Phisiology. Proceedings of the Twenty Eighth Annual Convention of the American Association of Equine Practitioners (AAEP). Atlanta, Georgia, p. 1520, dez., 1982.

COUROUCÉ, A.; CHATARD, J. C.; AUVINET, B. Estimation of Performance Potential of Standardbred Trotters from Blood Lactate Concentrations Measured in Field Conditions. Equine Veterinary Journal. v. 29, n. 5, p. 365-369, 1997.

DAVIE, A. J.; EVANS, D. L. Blood Lactate Responses to Submaximal Field Exercise Tests in Thoroughbred Horses. The Veterinary Journal, v. 159, p. 252-258, 2000.

EATON, M. D. Energetics and Performance-Structural Considerations in Equine Sports Medicine. In: HODGSON, D. R.; ROSE, R. J. (Ed.) The Athletic Horse. W. B. Saunders Company, 1994. cap. 4, p. 49-62.

EATON, M. D. ; HODGSON, D. R.; EVANS, D. L.; ROSE, R. J. Effects of Low and Moderate-Intensity Training on Metabolism Responses to Exercise in Thoroughbreds. Equine Exercise Phisiology (5), Equine Veterinary Journal. Suppl., v. 30, p. 521-527, 1999.

EVANS, D. L.; HARRIS, R. C.; SNOW, D. H. Correlation of Racing Performance with Blood Lactate and Heart Rate After Exercise in Thoroughbred Horses. Equine Veterinary Journal, v. 25, n. 5, p. 441445, 1993.

EVANS, D. L. Overview of Equine Exercise Physiology and Biochemistry. In: EVANS, D. L. (Ed.) Training and Fitness in Athletic Horses. Rural Industries Research and Development Corporation, 2000. cap. 2, p.1032.

FENGER, C. K.; McKEEVER,. K. H.; HINCHCLIFF, K. W.; KOHN, C. W. Determinants of Oxygen Delivery and Hemoglobin Saturation During Incremental Exercise in Horses. American Journal of Veterinary Research. v. 61, n. 10, p. 1325-1332, out, 2000.

GEOR, R. J.; McCUTCHEON, L. J.; SHEN, H. Muscular and Metabolic Responses to Moderate-Intensity Short-Term Training. Equine Exercise Physiology 5. Equine Veterinary Journal. Suppl. v. 30, p. 311-317, 1999.

HARRIS, P. A.; MARLIN, D. J.; GRAY, J. Plasma Aspartate Aminotransferase and Creatine Kinase Activities in Thoroughbred Racehorses in Relation to Age, Sex, Exercise and Training. The Veterinary Journal, v.155, p. 295-304, 1998.
As velocidades desenvolvidas na esteira podem ser semelhantes àquelas a que os animais desenvolvem nas corridas. Isto equivale a dizer que na esteira é possível desenvolver velocidade de rápida aceleração, a partir do momento em que está em inércia até uma velocidade de aproximadamente $15 \mathrm{~m} / \mathrm{s}$, para que seja possível simular condições similares àquelas estabelecidas em condições de corrida.

Observou-se também que os animais com melhor condicionamento físico apresentam menor elevação da atividade sérica de CK. Consequentemente, esta enzima pode ser utilizada como referência segura quando da avaliação do condicionamento físico de um determinado animal, desde que sejam estabelecidos padrões de variação fisiológica.

Pode-se recomendar como válido o teste em esteira ergométrica, com a finalidade de padronizar os valores bioquímicos nos cavalos de corridas, com vistas a uma avaliação mais confiável do seu condicionamento físico, levando-se ainda em consideração o fato de que o controle do monitoramento do animal costuma ser seguro e confiável durante as diferentes fases do teste.

LUNA, S. P. L. Equilíbrio Ácido-Básico. In: Apostila da Disciplina de Anestesiologia Veterinária do Curso de Medicina Veterinária da FMVZUNESP-Botucatu, 2002. p. 1-22.

MANOHAR, M.; GOETZ, T. E.; HASSAN, A. S. Effect of Prior HighIntensity Exercise-Induced Arterial Hypoxemia in Thoroughbred Horses. Journal of Applied Physiology, v. 90, n. 6, p. 2371-2377, jun. 2001.

MAYES, P. A. Bioenergética e metabolismo de carboidratos e lipídeos. In: MURRAY, R. K.; GRANNER, D. K.; MAYES, P. A.; RODWELL, V.W. Harper: Bioquímica. 8. ed. São Paulo: Atheneu, 1998. Seção II, cap.12, p. 109-291.

MILNE, D.W. Effects of Training on Biochemical Values in Standardbred Horses. American Journal of Veterinary Research. v. 37, n. 3, p. 285290, 1976.

MILNE, D. W. Biochemical Parameters for Assessment of Conditioning in the Horse. Proceedings of the Twenty Eighth Annual Convention of the American Association of Equine Practitioners (AAEP). Atlanta, Georgia, p. 49-53, dez. 1982.

MISUMI, K.; SAKAMOTO, H.; SHIMIZU, R. Changes in Blood Lactate and Heart Rate in Thoroughbred Horses During Swimming and Running According to Their Stage of Training. Veterinary Record, v. 135, n. 3, (3), p. 226-228, set. 1994.

MORRIS, E. Assessment of Performance Problems - Fitness Testing. In: ROBINSON, E. (Ed.) Current Therapy in Equine Medicine 3. W. B. Saunders Company, 1992. Seção 19, p. 802-806.

RAINGER, J. E.; EVANS, D. L.; HODGSON, D. R.; ROSE, R. J. Blood Lactate Disappearance After Maximal Exercise in Trained and Detrained Horses. Research in Veterinary Science, v. 57, p. 325-331, 1994.

RAINGER. J. E.; EVANS, D. L.; HODGSON, D. R.; ROSE, R. J. Distribution of Lactate in Plasma and Erythrocytes During and After Exercise in Horses. British Veterinary Journal, v. 151, p. 299-310, 1995.

RODRIGUES, P. C. Teste de Hipóteses. In: Bioestatística. 2. ed. Niterói: EdUFF 1993, cap. 12, p. 79-88.

ROSE, R.J. Problems of the Performance and Endurance Horse - Poor Performance Syndrome: Investigation and DiagnosticTechniques. In: ROBINSON, E. (Ed.) Current Therapy in Equine Medicine 2. W. B. Saunders Company. seção 12, p. 469-474.

ROSE, R.J. Problems of the Performance and Endurance Horse - Fluid, Electrolyte and Acid-Base Disturbances Associated with Exercise. In: ROBINSON, E. (Ed.) Current Therapy in Equine Medicine 2. W. B. Saunders Company. seção 12, p. 479-481. 
ROSE, R. J.; HODGSON, D.R. Practical Considerations in Equine Sports Medicine - Clinical Exercise Testing. In: The Athletic Horse. W. B. Saunders Company, 1994. 497 p., seção 2, cap.12, p. 245-258.

ROSE, B.D.; POST, T.W. Regulation of Water and Electrolyte Balance Regulation of Acid-Base Balance. In: Clinical Physiology of Acid-Base and Eletrolyte Disorders. 5. ed. McGraw-Hill, 2001. 992 p, seção 2, cap. 11, p. 239-402.

SEEHERMAN, H.J.; MORRIS, E.A. Application of a Standardised Treadmill Exercise Test for Clinical Evaluation of Fitness in 10 Thoroughbred Racehorses. Equine Veterinary Journal, n. 9, p. 26-34, jun. 1990.

SNOW, D.H.; MACKENZIE, G. Some Metabolic Effects of Maximal Exercise in the Horse and Adaptations with Training. Equine Veterinary Journal, v. 9, n. 3, p. 134-140, 1977.
TOLEDO, P.S.; JÚNIOR, M.D.; FERNANDES, W.R.;MAGONE, M. Atividade Sérica de Aspartato Aminotranferase, Creatina Quinase, GamaGlutamiltranferase, Lactato Desidrogenase e Glicemia de Cavalos da Raça P.S.I. Submetidos a Exercícios de Diferentes Intensidades. Revista Brasileira de Ciência Veterinária, v. 8, n.2, p. 73-77, maio/ago. 2001.

TYLER-McGOWAN, C. M.; GOLLAND, L. C.; EVANS, D. L.; HODGSON, D. R.; ROSE, R.J. Haematological and Biochemical Responses to Training and Overtraining. Equine Exercise Physiology 5. Equine Veterinary Journal. Suppl. 30, p. 621-625, 1999.

VALETTE, J. P.; BARREY, E.; AUVINET, B.; GALLOUX, P.; WOLTER, R. Exercise Tests in Saddle Horses. 2: The Kinetics of Blood Lactate During Constant Exercise Tests on a Treadmill. Journal of Equine Veterinary Science, v. 13, n. 8, p. 465-468, 1993. 